\title{
Session 3
}

\section{Star Formation Processes and Feedback}




\title{
Star Formation Through Cosmic Time
}

\author{
Michael A. Dopita \\ ${ }^{2}$ Research School of Astronomy \& Astrophysics, Australian National University, \\ Mt. Stromlo Observatory, Cotter Road, Weston Creek, ACT 2611, Australia \\ email: Michael.Dopita@anu.edu.au
}

\begin{abstract}
This paper reviews the star formation history of the Universe, from the first stars to the current day, with emphasis on the critical analysis of the techniques that have been used to determine it, especially considering the role of dust. We consider the first population of stars, the Population III stars, were formed at redshifts ranging as high as $z \sim 60$, the formation of the Globular Clusters, the main epoch of galaxy formation. In the sub-mm galaxies and high-redshift radio galaxies the collapse of massive galaxies was surprisingly rapid, and that the growth of super-massive black holes at their centers provides the energy input to eject the galactic interstellar medium while at the same time precipitating a final burst of star formation and the ejection of their ISM so that the subsequent evolution of these galaxies is passive.
\end{abstract}

Keywords. Physical Processes: neutrinos, shock waves, ISM: dust, HII regions, Galaxies: formation, evolution, high-redshift, jets

\section{The First Stars}

The Population III stars are probably described by a top-heavy IMF, a consequence of $\mathrm{H}_{2}$ cooling. Although it has been generally assumed that these formed at redshift $z \sim 20$ e.g. Yoshida et al. (2003), they may have been formed as little as $\sim 30$ Myr after the Big Bang corresponding to $z \sim 65$; Naoz, Noter \& Barkana (2006). At this time the overdense regions contained about $10^{5} M_{\odot}$. However, it is not until $\sim 500 \mathrm{Myr}$ after the Big Bang $(z \sim 12)$ that systems as large as the Milky Way start to assemble. These first stars are probably not sufficient of themselves to re-ionize the Universe, but they can locally ionize the medium, and on going supernova serve the important role of polluting it fairly extensively with heavy elements, which allows the next generation stars to form more easily. Sophisticated hydrodynamical simulations of this process have been made by Yoshida (2006).

The second generation of stars should have a more "normal" IMF and will assemble in the intermediate mass condensations which have sometimes been referred to as "minihalos" $\left(10^{7}-10^{10} \mathrm{M}_{\odot}\right)$. It is these stars (discussed further in the next section) which are the best candidates for the re-ionization of the Universe, which, by radically increasing the Jeans Mass of the gravitationally unstable sub-condensations, is then expected to suppress star formation until more normal galactic objects are formed.

Can we directly detect the first stars? This is one of the most exciting challenges of modern astrophysics. A variety of techniques have been suggested, and even utilized;

- By their Neutrino flux. Because of their redshift, these should appear as an excess at low energy $\sim 1.0 \mathrm{MeV}$; Daigne et al. (2005), Nakazoto, Sumiyoshi \& Yamada (2005).

- By their contribution to the IR or sub-mm Background Radiation - they provide an excess in the region $\lambda \sim 1-4 \mu \mathrm{m} ;$ e.g. Salvaterra et al. (2006), Bock et al. (2006).

- By the analysis of the distribution of "long Gamma-Ray Bursts; Murakami et al. (2005). This technique is sensitive at redshifts of $4 \leqslant z \leqslant 12$, and uses the spectral peak energy-to-luminosity relation recently found by Yonetoku et al. (2004). 


\section{Globular Clusters and the Missing Satellite Problem}

Although the $\Lambda \mathrm{CDM}$ models successfully describe the formation of large-scale structure and of cluster formation, they are less successful on a galactic scale. There are two major problems, first, disks of galaxies formed in the simulations are too small (the angular momentum problem, Navarro \& Steinmetz (2000)), and second, they predict in excess of 100 satellite systems of our Galaxy, whilst only a handful are seen (this is generally referred to as the satellite problem). While the first issue may be influenced by numerical resolution, the second is more serious. It is possible that some (or many) of the satellite systems have been tidally disrupted or have merged with the bulge of the galaxy. A wide variety of possible galactic assembly histories are possible according to theoretical Nbody $\Lambda$ CDM modelling, Gill et al. (2004). In this work, those objects with an evolutionary history that best fit what we know about our own Galaxy display a high degree of satellite disruption. In the Galaxy, ancient objects are observed in about the correct numbers to account for the 'missing' satellites - the globular clusters. From their inferred age, their low metallicities, and their chemo-dynamic properties which match the bulge and halo components of the Galaxy, Venn et al. (2004), we know that these are representative of a population of objects formed during the initial collapse of the Galaxy.

The current-day stellar masses of the globular clusters $\left(\sim 10^{5}-10^{6} \mathrm{M}_{\odot}\right)$ are likely to be only a few percent (at most) of their primordial baryonic mass because of all the massloss process which have operated throughout cosmic time. Their ISM is ejected either as hot gas or as entrained winds or via interaction with the mass-loaded outflows. Stars are lost by tidal stripping and through dynamical relaxation, leaving only the tightly bound cores of the stellar cluster. The weakly-bound dark matter is very easily tidally stripped Gill et al. (2004) - and there is no evidence for dark matter in any globular cluster today. Thus, the Globular Clusters might possibly be considered as the 'ghosts' of the missing satellites. The initial mass of the proto-globular cluster may well have been as high as $10^{7}-10^{10} \mathrm{M}_{\odot}$. The low mass limit of the globular clusters today would be then determined by the smallest mass which is able to form a dense core of stars stable against the loss of their dark matter halo, their ISM, and both the dynamical relaxation and tidal stripping processes. The upper mass limit is determined by dynamical friction, which increases as the square of the satellite mass. The most massive proto-clusters will have merged into the bulge through dynamical friction. The physics of these dynamical and feedback processes have not been adequately modeled, but are currently being studied by Dopita \& Sutherland (2006, in preparation).

\section{Star Formation Indicators in the Epoch of Galaxy Formation}

Once galaxies form, we see star formation proceeding is a wide variety of environments, many of which are highly dust obscured. This makes the determination of the star formation rates difficult, and the complexities of this process are often underestimated by the observers who want to construct Lilly-Madau plots of the history of the star formation density of the Universe; Lilly et al. (1996), Madau et al. (1996). It is therefore appropriate to briefly review the techniques that are currently available which enable us to infer star formation rates in galaxies.

Almost any feature in the spectral energy distribution could, in principle, be used as a star formation rate (SFR) indicator, provided the appropriate bolometric correction can be computed. This is by no means a trivial exercise, and errors of factors of two or three are easily generated, both on the observational and the theoretical fronts. Here we briefly discuss some of the more commonly-used techniques to determine the SFR in galaxies. 
Others also exist (for example, the X-ray flux), but these are not yet sufficiently well understood from a theoretical point of view to warrant inclusion here.

The optical/UV continuum For these techniques the effects of dust obscuration in and around the star-forming regions are particularly severe. The dust forms a patchy screen in front of the hot stars, and so the absorption is not described by a standard extinction law, but rather by an attenuation law (Fischera et al. 2003; 2004). Provided that the reddening correction can be made, and the initial mass function (IMF) is invariant, the intrinsic UV luminosity should scale directly as the star formation rate Kennicutt (1998), Panuzzo et al. (2003): The far-IR continuum The use of the far-IR emission from warm dust associated with the star formation region may provide a more reliable estimate of the SFR (Dwek 1998; Gispert et al. 2000). SFRs derived in this way frequently assume that the dust is effectively acting as a re-processing bolometer wrapped around the star forming region, and that cool and old stars do not provide too much of a contribution. In any real galaxy, old stars exist, and the stars are neither unobscured by dust, and nor are they totally obscured Popescu et al. (2000). This degree of obscuration is one of the many "geometrical" factors which render the derivation of the absolute SFR quite uncertain (Inoue 2002; Dopita et al. 2005).

The dust obscuration is observed to increase both with galaxian mass Kewley et al. (2002), and with the absolute rate star formation (Buat et al. 1999; Adelberger \& Steidel 2000; Dopita et al. 20002; Vijh et al. 2002). This can be understood as the combination of the degree of chemical evolution of the interstellar medium, which leads to a low dust to gas ratio in dwarf galaxies, and of the operation of the Schmidt (1959) Law of star formation, which couples the star formation rate per unit area to the gas surface density, and therefore, for a given dust to gas ratio, to the dust obscuration. In turn, the star formation is coupled to the global rate of star formation by the Kennicutt (1998) relation; $\Sigma_{\mathrm{SFR}} \propto \Sigma_{\text {gas }}^{n}$, with $n \sim 1.3-1.6$.

The radio continuum The radio continuum has also been used as a star formation rate indicator. Observationally, there is an extraordinarily close correlation between the $60 \mu \mathrm{m}$ infrared continuum and the radio $1.4 \mathrm{GHz}$ continuum of star forming galaxies. This linear correlation spans $\sim 5$ decades of magnitude with less than 0.3 dex dispersion (Yun et al. 2001; Wunderlich et al. 1987). Somewhat remarkably, this relationship couples a purely thermal process with a non-thermal process, over many decades of flux, and locally, within individual galaxies.

Recombination emission lines A fairly direct and extensively-used technique is to measure hydrogen recombination line fluxes. Provided that the HII region can absorb all the EUV photons produced by the central stars, this should be a reliable technique, since in this case the flux in any hydrogen line is simply proportional to the number of photons produced by the hot stars, which is in turn proportional to the birthrate of massive stars. This relationship has been well calibrated at solar metallicity for the $\mathrm{H} \alpha$ line (Kennicutt 1998; Panuzzo et al. 2003). Provided that the star-formation regions are resolved, their Balmer decrements can be used to estimate the absorption in any foreground dust screen. However, it is possible that some star forming regions are completely obscured, even at $\mathrm{H} \alpha$. This problem can be avoided by observing at infrared wavelengths, in $\operatorname{Br} \alpha$, for example. A further complication is that the dust content of the nebula is metallicitydependent, and therefore an appreciable fraction of the hydrogen ionizing photons may be absorbed by dust in high metallicity HII regions (Dopita et al. 2003).

[OII] emission lines The Balmer lines shift out of the visible range by $z>0.4$. The other strong emission lines accessible at higher redshift are the [OII] and [OIII] lines. Of these, the [OIII] lines are known to be very sensitive to the ionization parameter, and therefore the [OII] $\lambda 3727,9 \AA$ lines have been used of necessity to try to estimate the SFR 


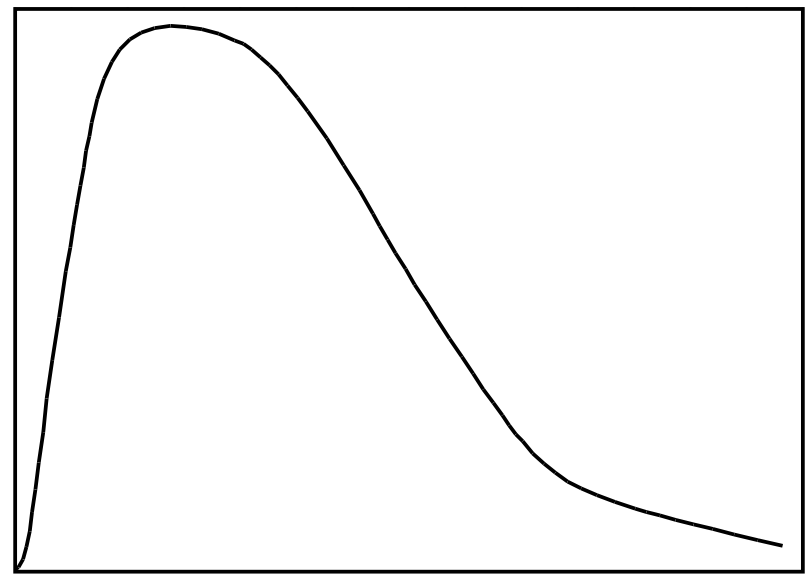

Figure 1. The Madau-Lilly diagram (approximately) transformed to physical parameters. Here a specific star formation of unity carried on throughout cosmic time would have ensured that all of the baryonic matter is incorporated in a star at some time. Note the plateau in star formation between roughly 2 and 4 Gyr, corresponding to the epoch of formation of the most massive galaxies, and the steep decline in star formation activities since that time.

for galaxies lying in the redshift range $z \sim 0.4-1.5$. Photoionization models show that these are by no means ideal as star formation indicators, because their strength not only depends on the attenuation, which tends to be large in the UV, but also on the metallicity of the ionized gas. These may lead to large discrepancies between SFR estimated from $\mathrm{H} \alpha$ and from the [OII] lines Charlot et al. (2002). However, Kewley and her collaborators Kewley et al. (2003) have developed techniques to allow corrections for both metallicity and reddening which bring the results of these two emission line techniques into much better agreement.

\section{The Madau-Lilly Plot}

The results of surveys of galaxies using one or more of the diagnostic tools outlined in the previous section is usually summarized in the form of a Madau - Lilly (or Lilly - Madau) plot, a plot of the star formation rate per unit co-moving volume of the Universe against redshift (Lilly et al. 1996; Madau et al. 1996). Apart from the uncertainty introduced by the variety of heterogeneous samples and techniques, there is considerable uncertainty attached to the intrinsic reddening corrections, especially at high redshift. This said, it is remarkable that a fair degree of convergence is reached, with various methods agreeing within a factor of three or so. For a recent complilation from the literature, see Fardal et al. (2006), and for $z<0.5$, see Hanish et al. (2006).

In order to demonstrate explicitly how star formation evolves with time, we have used the references cited above to generate a Madau - Lilly plot transformed to "physical" parameters (see Figure 1). This emphasizes the broad peak in star formation activity in the epoch of massive mergers and ultra-luminous starbursts, which correspond to the formation of the massive elliptical galaxies seen in the nearby universe. It also strikingly emphasizes the steep decline in star formation activity to the current epoch.

These estimates of the evolution of the star formation density should correctly predict the evolution of the stellar mass density of the Universe. Surprisingly, this appears to work rather well when the sample of galaxies is large enough. Gwyn \& Hartwick (2005) 
used NICMOS on the HST to determine this quantity from 796 galaxies in the Hubble Deep Field, and finds agreement to within the errors of the Madau - Lilly diagram itself.

\section{Sub-mm, Lyman $-\alpha$ and Hi-z Radio Galaxies}

In the peak of star formation shown in figure 1, most of the stars in the universe were formed. For the sub-mm galaxies (SMGs), Blain et al. (2004) has shown that, at a given dust temperature, the SMGs are typically 30 times as luminous as their ULIRG counterparts. Takagi et al.(2003a, 2003b) had previously found that most ULIRGS have a constant surface brightness of order $10^{12} L_{\odot} \mathrm{kpc}^{-2}$. Our own results, Dopita et al. (2005), indicate that this corresponds to an ISM with a pressure of order $P / k \sim 10^{7} \mathrm{~cm}^{-3} \mathrm{~K}$. These parameters probably characterise "maximal" star formation, above which gas is blown out into the halo of the galaxy and star formation quenched. Only mergers, which provide an additional ram-pressure confinement of the star formation activity may exceed this surface brightness. Thus, in order to scale the star formation up to the rates inferred for SMGs $\left(\sim 1000-5000 M_{\odot} \mathrm{yr}^{-1}\right)$, we must involve a greater area of the galaxy in star formation, rather than trying to cram more star formation into the same volume. For a typical value of $10^{13} L_{\odot} \mathrm{kpc}^{-2}$, we require "maximal" star formation over an area of $\sim 10 \mathrm{kpc}^{2}$, and the most luminous SMGs require star formation to be extended over an area of at order $\sim 100 \mathrm{kpc}^{2}$. We can conclude that SMGs are starbursts extended on a galaxy-wide scale, unlike the starburst regions seen in the nearby universe.

There is increasing observational evidence that this violent galaxy-wide star formation epoch is terminated by the feeding and growth of the massive black hole in such galaxies. Such evidence comes from the study of the high redshift radio galaxies (Hi-zRGs) with $z>3$ which rank among the most luminous, largest, and most massive galaxies known in the early Universe (De Breuck et al. 20002) and which are observed early in the Epoch of Galaxy Formation at a time when their super-massive black holes (SMBHs) are highly active, and while their relativistic jets are interacting most strongly on their host galaxies. Recent spectroscopy by Reuland et al. (2006) shows that the Lyman $-\alpha$ halos exhibit disturbed kinematics, with broad lines, large velocity shears, and expanding shells associated with the radio lobes. In addition, these halos halos are both chemically enriched by star formation and ionized throughout the majority of their volume. The relativistic jets drive strong shocks into the galactic medium and trigger shock-induced star formation on their peripheries.

These observations confirm the general physical idea of Silk \& Rees (1998) that feedback of the black hole on its host galaxy eventually limits the growth of the black hole. However unlike the Silk \& Rees (1998) concept, the feedback process is not primarily radiation pressure but is the mechanical energy input delivered by the relativistic jets. Thus, in the Hi-zRGs we observe the moment where galaxy collapse gives way to mass ejection, and a newly born galaxy is revealed. We may further speculate that this is the defining moment where the such galaxies enjoy one last violent burst of shock-induced star formation before beginning their evolution to become the "red and dead" massive Elliptical galaxies we see in our local universe.

\section{Acknowledgements}

M. Dopita acknowledges the support of both the Australian National University and of the Australian Research Council (ARC) through his ARC Australian Federation Fellowship, and through the ARC Discovery project grants DP0208445 \& DP0664434. 


\section{References}

Adelberger, K.L., \& Steidel, C.C. 2000, ApJ, 544, 218.

Blain, A. W., Chapman, S. C., Smail, I. \& Ivison, R. 2004, ApJ, 611, 52.

Bock et al. 2006 New. Ast. Rev, 50, 215-220.

Buat, V., Donas, J., Milliard, B. \& Xu, C. 1999, A\&A, 352, 371.

Charlot, S., et al. 2002, MNRAS, 330, 876.

Daigne, Olive, Sandick \& Vangioni 2005, Phys Rev. D 72, 103007.

De Breuck, C., et al. 2002, AJ, 123, 637.

Dopita, M. A., Pereira, M. Kewley, L. J. \& Capaccioli, M. 2002, ApJS, 143, 47.

Dopita, M. A., Groves, B. A., Sutherland, R. S., \& Kewley, L. J. 2003,ApJ, 583, 727.

Dopita, M. A., et al. 2005, ApJ, 619, 755.

Dwek, E., et al. 1998, ApJ, 508, 106.

Fardal, M. A., Katz, N., Weinberg, D. H. \& Davé, R. 2006, MNRAS (in press) astro-ph 0604534.

Fischera, J., Dopita, M. A., \& Sutherland, R. S., 2003, ApJL, 599, L21.

Fischera, J., Dopita, M. A. 2004, ApJ, 611, 919.

Gill, S. P. D., Knebe, A., Gibson, B. K., \& Dopita, M. A. 2004, MNRAS, 351, 410.

Gispert, R., Lagache, G., \& Puget, J. L. 2000, A\&A 360, 1.

Gwyn, S. D. J. \& Hartwick, F. D. A. 2005, AJ, 130, 1337.

Hanish, D. J. et al. 2006, ApJ, 649, 150.

Inoue, A. K. 2002, ApJ, 570, L97.

Kennicutt, R.C. Jr. 1998, Ann. Rev. A\&A, 36, 189.

Kewley, L. J., Geller, M. J., Jansen, R. A. \& Dopita, M. A. 2002, AJ, 124, 3135.

Kewley, L. J., Geller, M. J., \& Jansen, R. A., 2003, AJ, 127, 2002.

Lilly, S. J., Le Fevre, O., Hammer, F. \& Crampton, D. 1996, ApJL, 460, L1.

Madau, P., et al. 1996, MNRAS, 283, 138.

Murakami, T., et al. 2005, ApJ, 625, L13.

Nakazoto, K., Sumiyoshi, K. \& Yamada, S. 2005, astro-ph/0509868.

Naoz, S., Noter, S. \& Barkana, R. 2006, MNRAS (in press), astro-ph/0604050.

Navarro, J. F. \& Steinmetz, M. 2000, ApJ, 528, 607.

Panuzzo, P., Bressan, A., Granato, G. L., Silva, L. \& Danese, L. 2003, A\&A, 409, 99.

Popescu, C. C., Misiriotis, A., Kylafis, N. D., Tuffs, R. J., \& Fischera, J. 2000, A\&A, 362, 138.

Reuland, M. et al. 2006, ApJ (in press).

Salvaterra, R., Magliocchetti, M., Ferrara, A., \& Schneider, R. 2006, MNRAS 368, L6-9.

Schmidt, M. 1959, ApJ, 129, 243.

Silk, J. Rees, M. J. 1998, A\&A, 331, L1.

Takagi, T., Vansevius, V.. \& Arimoto, N. 2003, PASJ, 55, 385.

Takagi, T., Arimoto, N. \& Hanami, H. 2003, MNRAS, 340, 813.

Venn, K. A., Irwin, M., Shetrone, M. D., Tout, C.A., Hill, V., \& Tolstoy, E. 2004, AJ, 128, 1177.

Vijh, U. P., Witt, A. F. \& Gordon, K. D. 2003, ApJ, 587, 533.

Wunderlich, E., Wielebinski, R., \& Klein, U. 1987, A\&A Suppl., 69, 487.

Yonetoku, D. et al. 2004, ApJ, 609, 935.

Yoshida 2006 New Ast. Revs. 50, 19-23.

Yoshida, N., Sokasian, A., Henquist, L. \& Springel, V. 2003, ApJ, 598, 73.

Yun, M. S., Reddy, N. A., \& Condon, J. J. 2001, ApJ, 554, 803.

\section{Discussion}

Alice Shapley: How did you derive the size scales (large 10-100kpc ${ }^{2}$ ) for maximal starbursts for submm galaxies? How do you reconcile these with the significantly more compact sizes that Linda Tacconi reported based on CO observations? 
Mike Dopita: The objects I showed have star formation rates of $1000-5000 \mathrm{M}_{\odot} / \mathrm{yr}$, compared with the $150 \mathrm{M}_{\odot} / \mathrm{yr}$ in the Tacconi's sample. The area is derived from the "maximal" SFR giving a luminosity of order $10^{12} \mathrm{~L}_{\odot} / \mathrm{kpc}^{2}$, above which winds are driven from the galaxy. So a few $10^{13} \mathrm{~L}_{\odot}$ corresponds to a few tens of $\mathrm{kpc}^{2}$. 